\title{
BMJ Open Protocol for an implementation and realist evaluation of a new organisational model for primary care practices in the canton of Vaud, Switzerland
}

\author{
Muriel Schutz Leuthold (D) , ${ }^{1}$ Joelle Schwarz, ${ }^{1}$ Joachim Marti, ${ }^{2}$ \\ Clémence Perraudin, ${ }^{3}$ Catherine Hudon (D) , ${ }^{4}$ Isabelle Peytremann-Bridevaux (D) , \\ Nicolas Senn, ${ }^{1}$ Christine Cohidon ${ }^{1}$
}

To cite: Schutz Leuthold M, Schwarz J, Marti J, et al. Protocol for an implementation and realist evaluation of a new organisational model for primary care practices in the canton of Vaud, Switzerland. BMJ Open 2020;10:e040154. doi:10.1136/ bmjopen-2020-040154

- Prepublication history and additional materials for this paper is available online. To view these files, please visit the journal online (http://dx.doi. org/10.1136/bmjopen-2020040154).

NS and CC are joint senior authors.

Received 08 May 2020

Revised 02 November 2020 Accepted 18 November 2020

Check for updates

(C) Author(s) (or their employer(s)) 2020. Re-use permitted under CC BY-NC. No commercial re-use. See rights and permissions. Published by BMJ.

For numbered affiliations see end of article.

Correspondence to

Muriel Schutz Leuthold;

muriel.schutz@unisante.ch

\section{ABSTRACT}

Introduction Continuity of care, especially for patients with complex needs, is a major challenge for healthcare systems in many high-income countries, including Switzerland. Since 2015, a collaborative project between Unisanté-Department of Family Medicine (DMF), some general practitioners (GPs) and canton of Vaud's public health authorities has sought to develop a new organisational model for the provision of primary care to ensure better care coordination and to provide adapted care deliveries to patients' healthcare needs. The model's main component is the addition of a primary care nurse to GPs practices. Three additional tools are individualised patient care plans, electronic medical records and patient empanelment. To assess this model, a 2-year pilot study has begun in nine GPS' practices in the canton. This paper presents the protocol for an evaluation of the implementation and effectiveness of the new organisational model.

Method and analysis We will conduct a beforeand-after study using a mixed-methods and a realist approach. First, we will use quantitative and qualitative data to assess the new organisational model's implementation (feasibility, fidelity, acceptability and costs) and effectiveness (healthcare services use, patient experience, staff experience and patient-level costs). Combining this data with focus group data will enable a realist evaluation of the pilot project, which will help understand the elements of context and mechanism that affect implementation.

Ethics and dissemination The evaluation will inform the canton of Vaud's health authorities about the limits of and perspectives for this organisational model. All results will also be made available to the practices and the patients involved. At the end of the project, we will propose organisational adaptations and a sustainable financial model for extending the model to other practices in the canton and potentially to the national level.

The canton of Vaud's Human Research Ethics Committee approved the study, and Data Protection and Information Law Authority gave a favourable opinion concerning data processing procedures.
Strengths and limitations of this study

- The quasi-experimental design will estimate the causal impact of the addition of the primary care nurse (PCN) and PCN's activities to the practice's staff without randomisation.

- This comprehensive mixed-methods approach with a convergent design will provide complementary data and a holistic view of the implementation and functioning of the pilot implementation.

- The realist evaluation will answer questions that are not addressed by implementation evaluation: how, for whom, in what context and why the intervention works or does not work.

- The quantitative evaluation will be restricted to a before-and-after comparison, without a control group, that will limit interpretations of the effectiveness of outcomes.

- The limited sample size of patients with a care plan will limit the interpretation and understanding of quantitative data.

\section{INTRODUCTION}

Like many high-income countries, Switzerland's healthcare system is under the strain of an ageing population and increasing number of patients with chronic conditions. To address demographic, health and cost constraints, healthcare systems must aim to reduce the fragmented provision of healthcare and reinforce the provision of primary care, especially general medicine. ${ }^{1}$

One of the factors with the potential to contribute most to reducing the fragmentation of care, and thereby ensure the adequate continuity of care, is achieving good coordination, especially for patients with complex needs (eg, multimorbidity, psychosocial care). Indeed, many high-income countries are currently reviewing the organisation of their healthcare systems in order to provide better continuity and coordination of care, 
especially for the elderly and patients with chronic conditions. Besides the challenge of delivering appropriate care, these new organisational models also have significant impacts on costs. ${ }^{23}$ General practitioners (GPs) have excellent overall knowledge about each of their patients and their local community, and they are thus well positioned to oversee and address the challenge of the coordination of care. ${ }^{4}$

The new organisational models emerging around the world aim also to reinforce primary care medicine as a key community actor. They often integrate other healthcare professionals (eg, nurses, social workers), are associated with key new organisational approaches such as electronic medical records (EMRs), case management or individual care plans $^{56}$; they also imply the development of innovative financing mechanisms, such as bundled payments or pay-forperformance schemes. ${ }^{7}$

Switzerland's current organisational model for the provision of primary care via GPs'practices is not fully up to the current challenges facing its healthcare system. ${ }^{8}$ Many (45\%) GPs work single-handedly, with limited or no multidisciplinary collaboration. ${ }^{9}$ They work almost exclusively with medical assistants, ${ }^{8}$ who support them in administrative tasks and by administering simple clinical procedures. Moreover, the demography of GPs is evolving towards a potential shortage. ${ }^{10}$

Since 2015, Unisanté-Department of Family Medicine (DMF), some GPs' practices and canton of Vaud's public health authorities have been working on a unique collaborative project to develop a new organisational model for the provision of care in GPs' practices. Based on a literature review and a consultation process involving a large multiprofessional panel of experts, a conceptual model was designed involving the addition of a primary care nurse (PCN) to the staff of family medicine practices as its main component. Three other components were individualised care plans for case management activities, EMRs and patient empanelment. ${ }^{11}$ With the patients' empanelment, this new organisational model aims to provide the average number of patients followed by each GP and a global vision of health and demographic characteristics of these patients. Additionally, with the three other components it aims to provide more adapted deliveries to patients' healthcare needs and a better care coordination.

A new organisational model, based on these components, will be tested and monitored through a 2-year pilot project involving nine GPs' practices in the canton of Vaud. A pilot will begin between July 2019 and June 2020. With a view to potentially scaling-up this new organisational model and ensuring its sustainability, its real-world implementation will be evaluated. The present article describes the components of the protocol of an implementation, effectiveness and realist evaluation of this pilot project. The logical model of this intervention presented in figure 1 describes resources, activities of the new organisational model and outcomes expected.

This study's aim is to evaluate the implementation of a new, conceptual organisational model in the real-world context of Western Switzerland, in order to propose an adaptable, sustainable organisation for the future. The study's specific objectives are to: (1) evaluate the new organisational model's implementation in terms of feasibility, fidelity, acceptability and costs; (2) evaluate the intervention's effectiveness (eg, staff and patient experience, healthcare services use) and (3) understand how the elements of context and mechanism affect the implementation.

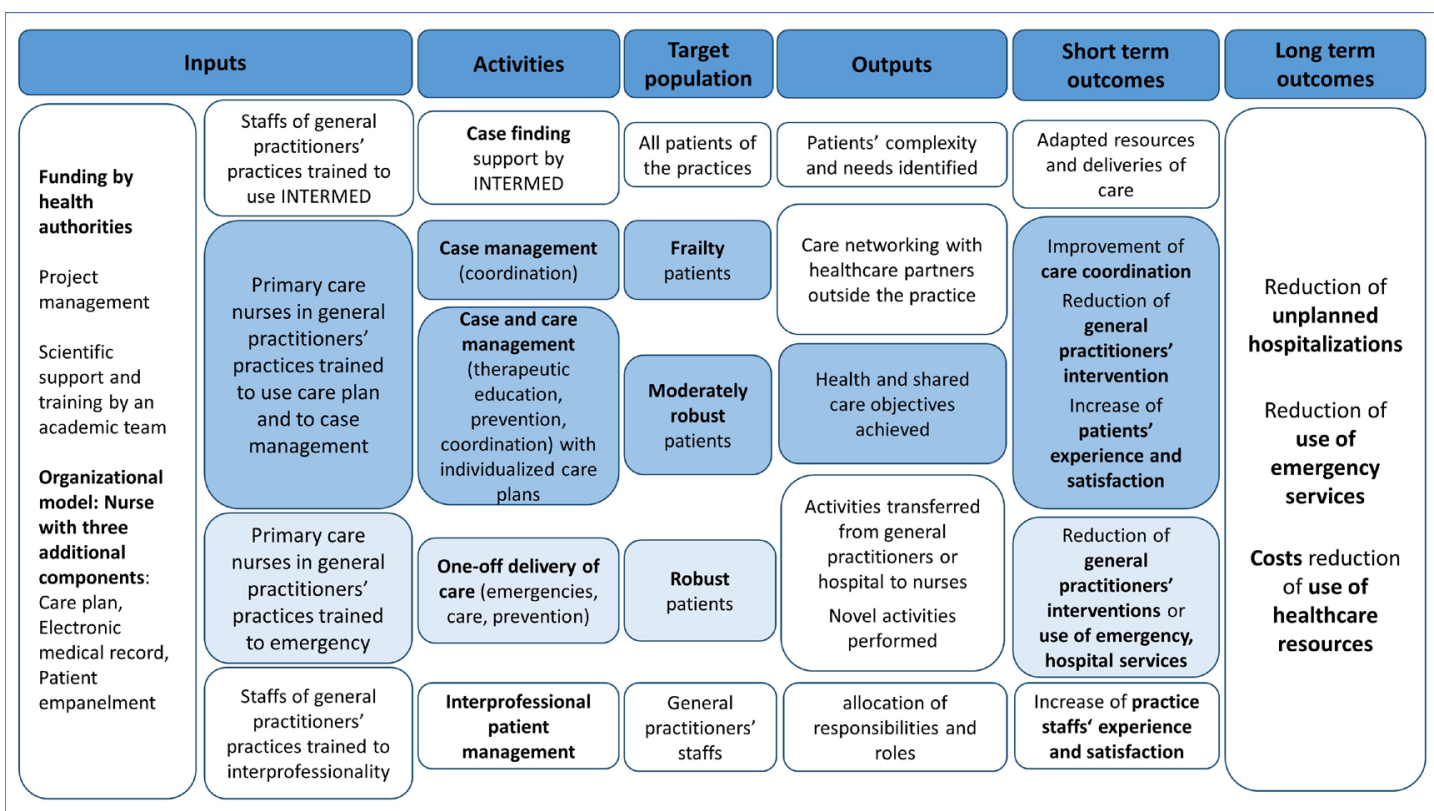

Figure 1 Logical model of the new organisational model. 
T0: Pre-implementation phase

T1: Start of implementation phase

T2: 12 months after start of

implementation

T3: 24 months after start of

implementation

Figure 2 Overall evaluation design.

\section{METHODS AND ANALYSIS}

\section{Patient and public involvement}

Patients and public were not involved in the development of the evaluation protocol.

\section{Study design}

We will use a quasi-experimental, mixed-method, beforeand-after study design, to assess the pilot project's processes and outcomes, as presented in figure 2. Evaluating complex organisational interventions such as this one is difficult because they target multiple interacting and evolving components at different organisational levels. Methods other than randomised controlled trials are better suited to comprehensively describing the benefits and limitations of such a new organisational model. ${ }^{12-15}$ Realist evaluation is particularly suited for describing what works, for whom, in which circumstances, and why, with the background intention of understanding whether and how interventions might be replicated in other contexts. ${ }^{16-19}$

The overall design process of the evaluation is composed of 10 steps. Steps 1-3 focus on the development of the new organisational model itself (previously done). Indeed, evaluation begins in the preimplementation phase (T0). Data collection occurs during preimplementation (step 4) and the implementation phase (T1) (step 5) over 2 years (step 6). After 1 year of implementation (T2), we will conduct a first analysis of the implementation and outcomes (step 7). This will be repeated after the second year of implementation (T3). At the end of the pilot project, a realist evaluation (step 8) will enable us to propose adaptations to the organisation tested (step 9 ). The evaluation's last step enables final recommendations about the new organisational model's sustainability to be proposed (step 10).

\section{Setting and population}

The pilot study will take place in nine GPs' practices (20 FTE GPs) in the Canton of Vaud, the most populous French-speaking canton of Switzerland, with approximatively 800000 inhabitants and 900 GPs working in either single-handedly or group private practices.

The selection criteria for the practices included interest for the project, localisation (urban and rural areas) and workforce (number of physicians, medical assistants).

All primary care practice staff members (PCNs, GPs and medical assistants) will be involved in interventions. They will all have the possibility to participate in focus groups and interviews to assess implementation outcomes and to refine and validate what works, for whom, and in which circumstances. All patients visiting the practice during its 2-year evaluation period will be eligible for participation in the interventions, except those under 18 years old or with severe cognitive disorders.

\section{Preimplementation}

In conjunction with the DMF, GPs from each participating practice will recruit a nurse. Because there is no specific training for nurses to work in GPs' practices in Switzerland, nurses will be recruited according to their professional experience and adequacy for each practice's needs.

Nevertheless, before starting their new jobs in GPs' practices, the nurses will undergo a 3-day training programme in enhancing their roles as PCNs. The training programme was developed based on indispensable prerequisites to make the new organisational model work and the needs identified from the nurses recruited for the pilot project. The following table 1 presents the content of the training and peoples involved in teaching. 


\begin{tabular}{lll} 
Table 1 & Training programme & \\
Content & $\begin{array}{l}\text { Duration } \\
\text { (hours) }\end{array}$ & Teacher \\
\hline $\begin{array}{l}\text { Pilot project MOCCA } \\
\text { and components } \\
\text { Case management }\end{array}$ & 3 & $\begin{array}{l}\text { Project manager of the } \\
\text { pilot project (DMF) }\end{array}$ \\
$\begin{array}{l}\text { Evaluation of the pilot } \\
\text { project }\end{array}$ & 4 & Research staff (DMF) \\
$\begin{array}{l}\text { Emergency response } \\
\text { Care plan }\end{array}$ & 1 & $\begin{array}{l}\text { General practitioner } \\
\text { (DMF) }\end{array}$ \\
\hline $\begin{array}{l}\text { Interprofessionality } \\
\text { Nurse, integrate care } \\
\text { specialist }\end{array}$ \\
\hline
\end{tabular}

During this preimplementation phase, the DMF will also ensure that the three additional components of the organisation model are available within the practices. Every practices recruited in the pilot project use already an EMR. However, a care plan for care coordination and a patient empanelment will be made available by the DMF.

\section{Intervention}

Once the nurse and the practice's staff trained, the PCN will deliver care to all types of patients, either alone or jointly with a GP or a medical assistant. To ensure that the nursing activities are better adapted to patients' healthcare needs, the medical assistants will update the patient empanelment and will administer the INTERMED SelfAssessment questionnaire (IMSA) to the patients. ${ }^{20-22}$ Hence, the range and intensity of a PCN's activities will vary according to patients' healthcare needs. They can be described and grouped in three categories as presented in figure 3, adapted from the Kaiser Permanente Triangle of Care. ${ }^{23}$

- For robust, independent patients, PCNs' interventions are one-offs (eg, focused on the management of small emergencies, benign infectious syndromes or

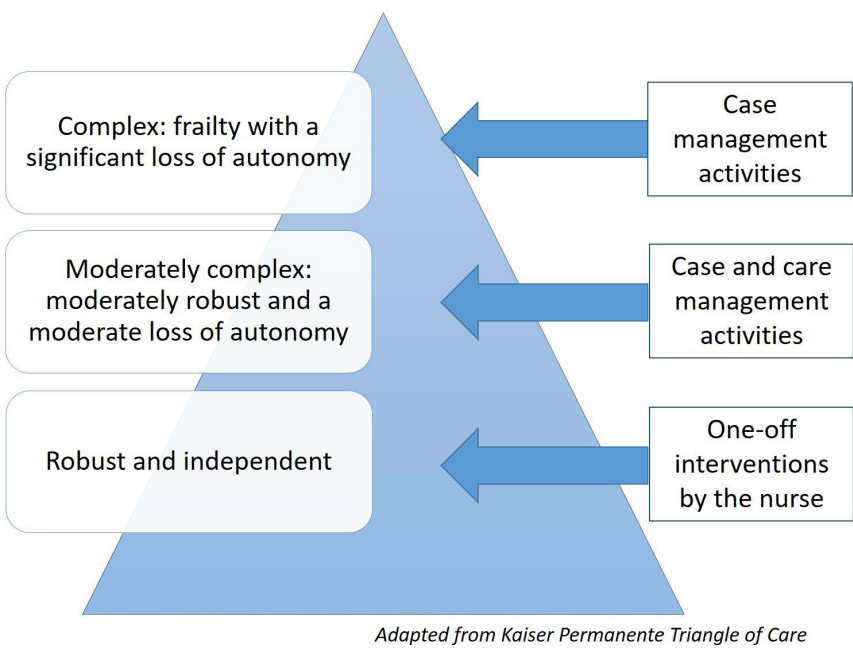

Figure 3 Primary care nurse interventions according to patients' levels of complexity. traumatology). At the GP's request, the PCNs will also intervene over a short period of time to treat acute health problems or to make primary prevention (eg, tobacco, diet).

- The greatest part of a PCN's activities will be dedicated to case and care management using individualised care plans (eg, therapeutic education, self-empowerment, secondary prevention and care coordination). The patients involved will have moderately complex health statuses, as shown in the middle of the pyramid (figure 3). Patients from this category are those with one or more chronic diseases at risk of deterioration of health status or complication. They will be identified according to GPs' medical opinion supported by a questionnaire to assess their biopsychosocial complexity and healthcare needs, that is, the IMSA questionnaire. ${ }^{20-22}$

- For complex patients with a significant loss of autonomy, PCNs will support GPs in coordinating care with healthcare partners outside the practice. This category of patients often benefits from the support of a large care network outside the practice, such as home care or specialised care. Examples include patients treated for cancer who have interdisciplinary care in oncology or elderly patients who benefit from home care by a nurse. The identification of these patients will be done in the same way as for the previous category.

PCNs will also propose health promotion, prevention, and clinical care activities (eg, wound care or drug administration), to all patients, whatever their level of complexity.

\section{Objective 1: implementation evaluation: process outcomes} As suggested in the literature, ${ }^{12}{ }^{24}$ implementation evaluation will be assessed in terms of the feasibility of the new organisational model, defined as the extent to which the intervention could be carried out in the Canton de Vaud's GPs' practices. We will also assess the fidelity of the implemented intervention with regard to the original protocol and the acceptability of the organisation in practices, referring to professionals' perceptions of whether the intervention has been satisfactory. Barriers and facilitators will be discussed with all the practice's professionals during individual interviews and focus groups with practice staff (GPs, PCNs, medical assistants). Finally, the intervention will be evaluated in terms of costs generated by the new organisation. Supplementary costs generated by the pilot study will be estimated and assessed in both the preimplementation and functioning phases. ${ }^{24}$

\section{Data collection}

In order to appropriately assess our first objective, several data collection approaches must be combined. We will use two types of quantitative and qualitative data: routine data and ad hoc data. A summary of the data collection strategy is presented in table 2 . 
Table 2 Data collection strategy for the implementation evaluation

\begin{tabular}{|c|c|c|c|c|}
\hline \multirow[b]{2}{*}{ Outcomes } & \multirow[b]{2}{*}{$\begin{array}{l}\text { Type of } \\
\text { data }\end{array}$} & \multirow[b]{2}{*}{$\begin{array}{l}\text { Sources and } \\
\text { format }\end{array}$} & \multicolumn{2}{|c|}{ Data collection } \\
\hline & & & $\begin{array}{l}\text { Routine } \\
\text { data }\end{array}$ & $\begin{array}{l}\text { Ad hoc } \\
\text { data }\end{array}$ \\
\hline \multirow[t]{5}{*}{ Feasibility } & Mixed & Application & & $x$ \\
\hline & & $\begin{array}{l}\text { Electronic } \\
\text { medical records }\end{array}$ & $x$ & \\
\hline & & Questionnaires & & $x$ \\
\hline & & $\begin{array}{l}\text { Non-participant } \\
\text { observations }\end{array}$ & & $x$ \\
\hline & & $\begin{array}{l}\text { Diaries (PCNs, } \\
\text { PM) }\end{array}$ & & $x$ \\
\hline \multirow[t]{5}{*}{ Fidelity } & Mixed & Application & & $x$ \\
\hline & & $\begin{array}{l}\text { Electronic } \\
\text { medical records }\end{array}$ & $x$ & \\
\hline & & Questionnaires & & $x$ \\
\hline & & $\begin{array}{l}\text { Non-participant } \\
\text { observations }\end{array}$ & & $x$ \\
\hline & & $\begin{array}{l}\text { Diaries (PCNs, } \\
\text { PM) }\end{array}$ & & $x$ \\
\hline \multirow[t]{2}{*}{ Acceptability } & Qualitative & $\begin{array}{l}\text { Interviews } \\
\text { (PCNs) }\end{array}$ & & $x$ \\
\hline & & $\begin{array}{l}\text { Focus groups } \\
\text { (GPs, medical } \\
\text { assistant) }\end{array}$ & & $x$ \\
\hline \multirow[t]{2}{*}{ Costs } & Quantitative & Application & & $x$ \\
\hline & & Questionnaires & & $x$ \\
\hline
\end{tabular}

GP, general practitioner; PCNs, primary care nurses; PM, project manager.

Routine data is the data that will be generated by practices in their day-to-day operation of the intervention, such as data from EMRs, individualised care plans, and patient empanelment. Indeed, these will be the principal sources of data.

Ad hoc data collection will involve the use of several sources and tools. The two main sources of quantitative data will be an electronic application and questionnaires. The web electronic application (on HTTPS, Hypertext Preprocessor (PHP) framework Laravel) is a tool especially developed to collect PCNs' activities throughout the pilot study. It is designed in form of list of activities, previously established by the GPs and PCNs involved in the pilot project. PCNs will selected the activity performed, its duration and the tariff heading. Most questionnaires will be administered using Research Electronic Data Capture (REDCap) software. Individual interviews with PCN's and separated focus group interviews with GPs and medical assistants and periodic non-participant observations will allow qualitative data collection. These qualitative data are collected electronically (diaries) or audio-recorded (interviews). Audio recordings will be transcribed, anonymised and stored on the DMF server.
All data (quantitative and qualitative) will be stored on the DMF server with access restricted to the investigation team.

The different measurement instruments and tools used for collecting data for the implementation evaluation are presented below and listed according to their relation with the respective outcomes.

\section{Feasibility}

To assess feasibility, we will evaluate the following aspects of the intervention:

- Practice organisation will be assessed using data on the number of patients affiliated to the practice, followed by the PCN using a care plan, refusing follow-up, and who cannot be followed-up due to saturation. Data will be collected from EMRs.

- PCN's delivery of care for patients with and without care plans, with detailed activities and duration, will be collected using an electronic application especially developed to identify PCNs' activities.

- Information regarding the practice organisationnumber of meetings, time taken to elaborate care plans-will also be collected in the electronic application.

- Practice structure, including human and material resources (number of GPs, medical assistants and PCNs, number of patients affiliated to the practice, use of EMRs, use of care plans and electronic patient lists), will be assessed using a paper questionnaire administered by the project manager (PM) during preimplementation.

- The resources and time required to adapt to and implement the new model of practice organisation, such as the number, duration of meetings and professionals involved will be collected by the PM using REDcap software. ${ }^{25}$

- Qualitative data from the PM's diary will inform aspects of practice organisation, including practice dynamics, challenges and motivation for participation in the pilot project.

\section{Fidelity}

Concerning fidelity, the main aspects observed will be:

- Information on PCNs' delivery of care to patients, with and without care plans, collected from electronic application, in terms of the type of activities carried out and their duration.

- Characteristics of patients followed by PGNs-such as sociodemographic data, IMSA scores, number of drugs prescribed, number of pathologies-will be obtained from EMRs.

- The division of tasks among practice staff will be assessed using data from EMRs and the electronic application.

- Qualitative data regarding practices' use of care plans (how care plans are established and negotiated between healthcare staff and patients) will be obtained from periodic non-participant observations by investigators and interviews with PCNs. 
- Qualitative data on aspects concerning practice organisation and dynamics, challenges, adaptive processes, and modifications to organisation will be obtained from individual interviews and focus groups with practice staff (GPs, PCNs, medical assistants).

\section{Acceptability}

Acceptability will be especially discussed with regards to the:

- Human and material resources used.

- Organisation and delegation of tasks.

- Functioning within practices.

- Interprofessional collaboration and communication within staffs' practices.

Acceptability will also be evaluated from patients' perspectives, using interviews and the rate of refusal of nursing follow-up entered in EMRs.

All of these interviews and focus groups will be performed by a sociologist with data-driven interview guides.

\section{Costs}

Costs will include:

- Costs of equipment, such as adapting EMRs for patient empanelment and materials for nursing activities (eg, office consumables, hardware) will be assessed using the paper questionnaire administered by the PM during the preimplementation phase.

- Human resources necessary for the preimplementation and implementation phases, measured in terms of the number of professionals involved (PCNs, medical assistants, GPs) and the number and duration of meetings within each practice for establishing and making the new organisational model work. These data will be collected by the PM using REDCap software and by the PCNs using the electronic application.

- Time and material required for training staff and supporting the project (number and duration of meetings, telephone calls and emails involving the practice and the PM) will also be collected by the PM using REDCap software and by PCNs using the electronic application.

\section{Data analysis}

To describe patient populations and GPs' practices, we will use standard descriptive statistics such as means, medians, SD and frequencies. The software used for these analyses will be StataSE V.16.

Qualitative data from interviews and focus groups will be coded and analysed, using an inductive approach to extract the main themes and subthemes. ${ }^{26}{ }^{27}$ Theme categories will then be discussed and refined by the investigators. Analyses will be performed using MAXQDA software (V.18).

Mixed-method analyses will be performed using a convergent design, integrating both quantitative and qualitative data. Hence, qualitative and quantitative results will be compared side by side in a joint table. ${ }^{28}$
The cost implications of the new organisational model will be assessed using a comprehensive microcosting approach. ${ }^{29}$ Pilot study data will allow us to estimate implementation costs, including the costs of equipment and human resources. For workforce-related costs, activities and their duration collected in the electronic application and REDcap software will be assigned their appropriate unit costs (ie, wage). Moreover, detailed data collected on care provision activities will allow us to measure the gap between what is reimbursed under current payment mechanisms (ie, mainly fee-for-service payments for medical and care activities) and which activities are performed but not reimbursed (or given a monetary value). These estimates, along with the assessment of the potential benefits revealed by the pilot project intervention, will allow us to suggest better-suited modes of remuneration. Finally, we will evaluate the short-term financial impact of the new organisational model from the practice perspective. ${ }^{30}$

\section{Objective 2: effectiveness evaluation: staff-level and patient- level outcomes}

Outcomes at the staff and patient levels will be evaluated in terms of the project's impact on the staff's experiences, patients' experiences, healthcare service use and costs to patients.

\section{Data collection}

In order to assess staff and patient-level outcomes, we will use an identical approach to the first objective, as presented in table 3 . Indeed, the measurement instruments and tools used will be the same with the exception of insurance reports. They are listed below for each outcomes with the corresponding variables.

\section{The new organisational model's impact on staff's experience}

At the practice level, we will use a self-administered questionnaire to evaluate the new organisational model's impact on the staff's experience in relation to the atmosphere among the team and staffs' satisfaction before

Table 3 Data collection strategy for the effectiveness evaluation

\begin{tabular}{|c|c|c|c|c|}
\hline \multirow[b]{2}{*}{ Outcomes } & \multirow[b]{2}{*}{$\begin{array}{l}\text { Type of } \\
\text { data }\end{array}$} & \multirow[b]{2}{*}{$\begin{array}{l}\text { Sources and } \\
\text { format }\end{array}$} & \multicolumn{2}{|c|}{ Data collection } \\
\hline & & & $\begin{array}{l}\text { Routine } \\
\text { data }\end{array}$ & $\begin{array}{l}\text { Ad hoc } \\
\text { data }\end{array}$ \\
\hline \multirow{3}{*}{$\begin{array}{l}\text { Staff's } \\
\text { experiences }\end{array}$} & \multirow[t]{3}{*}{ Mixed } & Questionnaires & & $x$ \\
\hline & & Interviews & & $x$ \\
\hline & & Focus groups & & $x$ \\
\hline \multirow{2}{*}{$\begin{array}{l}\text { Patients' } \\
\text { experiences }\end{array}$} & \multirow[t]{2}{*}{ Mixed } & Questionnaires & & $x$ \\
\hline & & Interview & & $x$ \\
\hline \multirow{3}{*}{$\begin{array}{l}\text { Health } \\
\text { services use } \\
\text { and patient- } \\
\text { level costs }\end{array}$} & \multirow[t]{3}{*}{ Quantitative } & Questionnaires & & $x$ \\
\hline & & $\begin{array}{l}\text { Electronic } \\
\text { medical records }\end{array}$ & \multirow[t]{2}{*}{$x$} & \\
\hline & & $\begin{array}{l}\text { Insurance } \\
\text { reports }\end{array}$ & & $x$ \\
\hline
\end{tabular}


and after the integration of PCNs. This questionnaire was developed from two previously validated tools used in the context of general practices. We will use the French version of the Team Climate Inventory questionnaire, validated by Beaulieu et al, to measure team climate. ${ }^{31}$ To assess staffs' satisfaction, we have selected one dimension of the questionnaire developed by Warr et $a l^{32}$

We will also compare team climate and staffs' satisfaction in nine voluntary control practices with similar original characteristics.

\section{The new organisational model's impact on patients' healthcare services use}

The intervention's impact or effect on the healthcare use of patients with a care plan and followed by a PCN will be measured twelve months before and twelve months after nursing follow-up. Via patients' care plans, annual healthcare assurance reports, and their self-administered questionnaire, the patient's experiences will be assessed by collecting the following data:

- Number and duration of unplanned hospitalisations.

- Number of emergency department visits.

- Number of consultations with the GP (at home and at the practice).

\section{The new organisational model's impact on patients' experience}

The aspects, detailed below, will be evaluated with regards to answers to a questionnaire self-administered before and after nursing follow-up:

- Accessibility in terms of delivery of care, timely access and first contact.

- Interprofessional communication with regard to shared decision-making and comprehensive medical care.

- Coordination and continuity of care.

- Provision of care in terms of goal-oriented care, health promotion activities.

- Support for patient empowerment.

The questionnaire was created from the validated French versions of the three following tools: the population questionnaire (Q.pop) ${ }^{33}$ the Patient Experience of Integrated Care Scale ${ }^{34}$ and the Person-Centered Primary Care Measure. ${ }^{35}$

\section{The new organisational model's impact on costs at patients' level}

The data collected to assess patients' evolving healthcare use following the implementation of the new organisational model will also enable us to measure the evolving use of healthcare resources and their costs, from patients' perspectives.

\section{Data Analysis}

Bivariate analyses ( $\chi^{2}$ tests) will be performed to compare outcomes with regard to practice staff and patients, before and after implementation of the new organisational model and after patient follow-up by the PCNs. These analyses will show us the new organisational model's impact on healthcare service use, costs, and patients' and staff's experiences. The software used for these analyses will be StataSE V.16.

For mixed-method analyses, we will also use a convergent design, integrating both quantitative and qualitative data. As data from each practice becomes available, we will merge them to build a mixed metamatrix. This matrix will estimate the benefits of the new organisational model at the patient level, looking specifically at patients' experiences, healthcare services use and costs. ${ }^{36}$ In order to perform these analyses, we will use the qualitative data analysed to assess the first objective.

Patient-level data will also be used to measure healthcare resources use and costs from the practice's perspective and how these evolve following the implementation of the new organisational model. These patient-level costs will be assessed before and after implementation, and regression models will be used to study the main determinants of costs.

\section{Objective 3: realist evaluation}

To understand how the elements of context and mechanism affect the new organisational model's implementation and outcomes, the pilot project's context-mechanism-outcome (CMO) configurations will be subjected to a realist evaluation conducted as described by Pawson and Tilley. ${ }^{37}$ This is done in several stages: formulation of the initial pilot programme theory and middle-range theories; testing these theories using both quantitative and qualitative data; and theory refinement. ${ }^{1637}$ The results from this protocol objective will be reported following the RAMESES II reporting standards for realist evaluations. ${ }^{38}$

\section{Data collection}

We will use different types of data to perform the realist evaluation and establish the refined organisational model.

- The data collected to assess implementation and effectiveness (both quantitative and qualitative) will be extracted to build semipredictable patterns of CMOs specific to each GPs' practice. ${ }^{39}$ We will especially identify qualitative data regarding practices' contextual frameworks and specific conditions-those that favour the implementation of the new organisational model, the processes of adaption and modification to it, and the staff's and patients' experiences.

- Existing local and national data (literature and healthcare system information), as well as data collected during the preimplementation phase, will be used to design our initial theories for the realist evaluation, including the hypothesised elements (context and mechanisms) expected to influence the targeted outcomes in a causal relationship. Data from literature and healthcare system information will also be used to build semipredictable patterns of CMOs.

- Specific-data issues raised by the focus groups with the practice staffs, the policy-makers, the PM and the study's investigators will allow us to refine and validate 


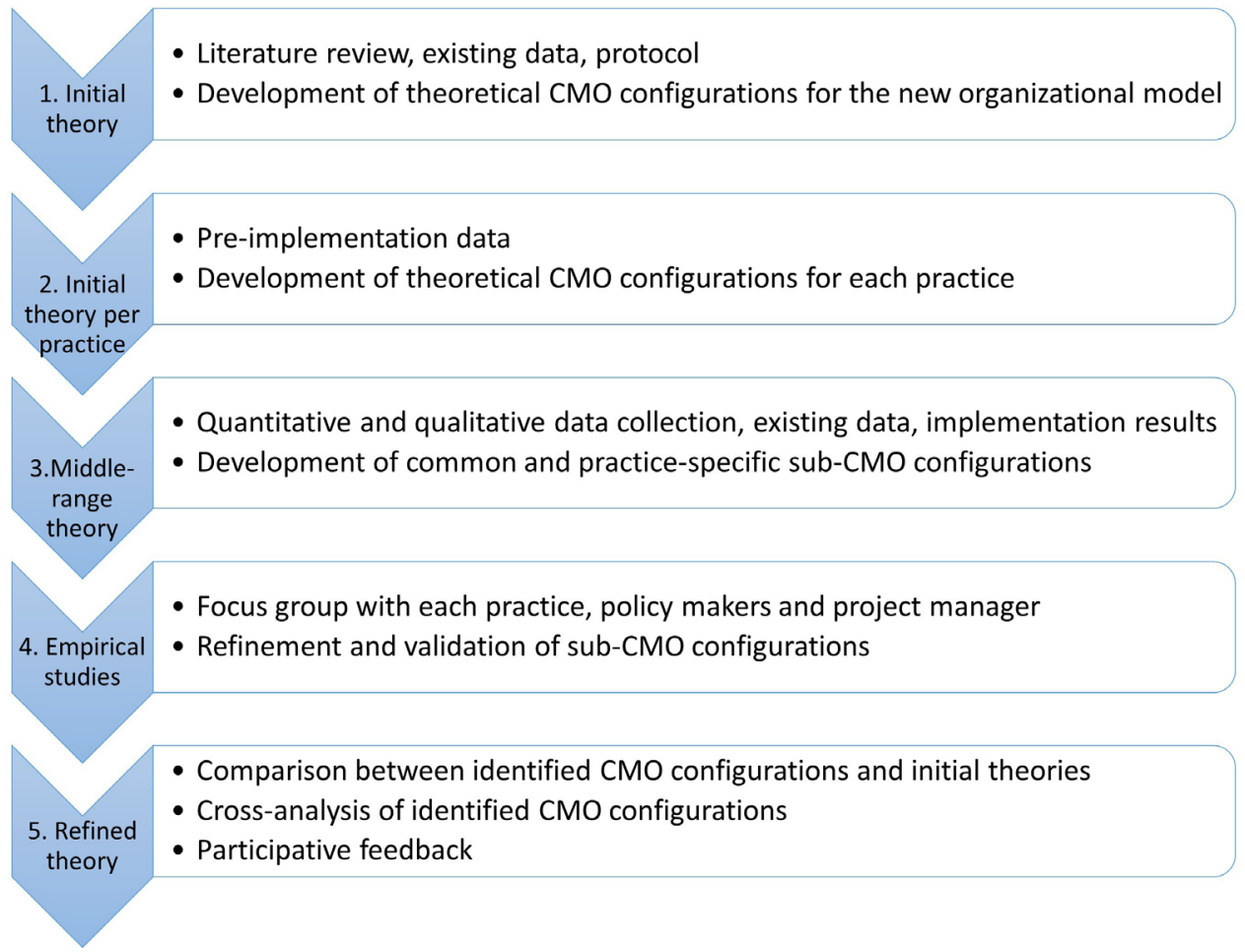

Figure 4 Steps in the realist evaluation.

the sub-CMOs for each practice, as well as the theories. ${ }^{40} 41$

\section{Data analysis}

The realist evaluation will be conducted in five steps (figure 4). Step 1 was the development of the initial theory of the conceptual organisational model, defined as following: Introducing a nurse in GPs' practices allow providing new delivery of care, such as one-off nursing delivery of care, care and case management $(\mathrm{M})$, with the aim of reducing healthcare services use, and costs to practices patients (E). The interprofessional collaboration within the practice and the development of new deliveries of care (M) and their impacts on the staff's experiences, patients' experiences, healthcare services use and costs to patients (E) are influenced by the local stetting, the organisation within the practice and individual factors (C)). This detailed how the intervention should produce its expected outcomes through a set of mechanisms and CMO configurations; it was derived from a literature review, the conceptual model's guidelines and existing local-level and national-level data. ${ }^{38}$

Step 2 will develop initial theories for each practice by identifying their specific contexts. Contexts will be studied at several levels: individual (nurses, GPs, medical assistants and patients), interpersonal (relationships, professional interactions inside practices), institutional (practices) and at the broader level, common to all nine practices, such as the sociohealth and political context in the canton of Vaud and Switzerland. ${ }^{42}$ Initial theories at the practice level will be completed with preimplementation data collected by the PM. The aim is to hypothesise on the causal links between context, mechanisms and targeted outcomes. Each CMO component will be thoroughly described.

Step 3 will involve configuring semi-predictable patterns of CMOs for each practice (middle-range theories) by extracting data from quantitative and qualitative data collected and from existing local and national data. We will also aim to identify how mechanisms can vary based on contextual elements (eg, practice setups or dynamics). Qualitative data will be coded using MAXQDA software via an analysis framework derived from the initial theory and the literature: participant (patients, practice staffs) descriptions; practices' components or strategies; proximal outcomes and their associated measures; the inner and outer contexts; and mechanisms triggered by the inner and outer contexts and how they might generate proximal and final outcomes. ${ }^{43}$ The middle-range theories (sub-CMOs) aim to explain why the organisational model works (or not) and has positive expected outcomes, and the specific ways in which it works (or not) in each specific practice. The refinement and validation of these middle-range theories will be derived via consensus discussion between the practice staffs (GPs, PCNs and medical assistants) and investigators about the synthesis and interpretation of findings.

As step 4, these theories will then be discussed with the practice staff in each practice, the PM and the policymakers in order to validate and refine their sub-CMOs.

Lastly, in step 5, we will first identify recurrent CMO configurations within each practice (case) $)^{37}$ and then across practices. This cross-case analysis will be used to 
refine the initial theory and to provide a deep understanding of how and why case management works (or not) in context.

\section{ETHICS AND DISSEMINATION}

This study is carried out in compliance with Swiss law. The project was submitted to the canton of Vaud's Human Research Ethics Committee (Req-2019-00544). The canton of Vaud's Data Protection and Information Law Authority gave a favourable opinion concerning data processing procedures. Information on the project will be given to each eligible patient, and they will be asked for their written consent to participate. Patients will have the opportunity to permit or refuse the use of coded healthcare data from their EMRs, to complete the primary care experience questionnaire and participate in interviews, non-participant observations and to give insurance reports.

This study represents a unique opportunity to assess the implementation and performance of a new organisational model for the primary care delivered via GPs' practices in the canton of Vaud, especially from the healthcare services and policy research perspectives. In other words, what are the broad, mid-to-long-term benefits and limitations to be expected for the canton's healthcare system and its patients? The before and after, mixed-methods approach used will assess whether this new organisational model has the potential to achieve its goals over the midto-long term, including improving the coordination and continuity of care. This will guide its potential generalisation to all of the practices in the canton of Vaud, and perhaps beyond. The project's results will be essential in order to inform and guide the canton's policymakers and healthcare authorities in any potential scaling-up of this organisational model. Results will be made available to policymakers, healthcare authorities, practices and patients.

\section{Author affiliations}

${ }^{1}$ Département Médecine de famille, Centre Universitaire de Médecine Générale et Santé Publique, Lausanne, Switzerland

${ }^{2}$ Département Epidémiologie et Systèmes de santé, Centre Universitaire de Médecine Générale et Santé Publique, Lausanne, Switzerland

${ }^{3}$ Département des Policliniques, Centre Universitaire de Médecine Générale et Santé Publique, Lausanne, Switzerland

${ }^{4}$ Département de Médecine de Famille et Médecine d'Urgence, Université de Sherbrooke, Sherbrooke, Quebec, Canada

Contributors MSL, CC and NS are the study's principal investigators. MSL, CC and NS conceived and planned the global design of the evaluation. MSL wrote the first and the final draft of the manuscript. JS contributed knowledge on qualitative research and realist evaluation. JM and CP contributed knowledge on costs evaluation. $\mathrm{CH}$ was involved for her expertise on the study subject and IP-B for her expertise in evaluation. All authors read, provided feedback and approved the final version of the protocol.

Funding The pilot is financially supported by the Service de la Santé Publique, Canton de Vaud.

Competing interests None declared.

Patient and public involvement Patients and/or the public were not involved in the design, or conduct, or reporting, or dissemination plans of this research.
Patient consent for publication Not required.

Provenance and peer review Not commissioned; externally peer reviewed.

Open access This is an open access article distributed in accordance with the Creative Commons Attribution Non Commercial (CC BY-NC 4.0) license, which permits others to distribute, remix, adapt, build upon this work non-commercially, and license their derivative works on different terms, provided the original work is properly cited, appropriate credit is given, any changes made indicated, and the use is non-commercial. See: http://creativecommons.org/licenses/by-nc/4.0/.

\section{ORCID iDs}

Muriel Schutz Leuthold http://orcid.org/0000-0001-8303-5126

Catherine Hudon http://orcid.org/0000-0001-6140-9916

Isabelle Peytremann-Bridevaux http://orcid.org/0000-0002-6514-8781

\section{REFERENCES}

1 Kringos DS, Boerma WGW, Hutchinson A, et al. Building primary care in a changing Europe. In: Policies EOohSa. Copenhagen, Denmark: European Observatory Health Policy Series, 2015.

2 Osborn R, Moulds D, Squires D, et al. International survey of older adults finds shortcomings in access, coordination, and patientcentered care. Health Aff 2014;33:2247-55.

3 Penm J, MacKinnon NJ, Strakowski SM, et al. Minding the gap: factors associated with primary care coordination of adults in 11 countries. Ann Fam Med 2017;15:113-9.

4 Stille CJ, Jerant A, Bell D, et al. Coordinating care across diseases, settings, and clinicians: a key role for the generalist in practice. Ann Intern Med 2005;142:700-8.

5 Edwards ST, Dorr DA, Landon BE. Can personalized care planning improve primary care? JAMA 2017;318:25

6 van Dongen JJJ, van Bokhoven MA, Daniëls R, et al. Developing interprofessional care plans in chronic care: a scoping review. BMC Fam Pract 2016;17:1-9.

7 de Bakker DH, Struijs JN, Baan CB, et al. Early results from adoption of bundled payment for diabetes care in the Netherlands show improvement in care coordination. Health Aff 2012;31:426-33.

8 Senn N, Ebert S, Cohidon C. La médecine de famille en Suisse perspectives. analyse sur La base des indicateurs Du programme spam (Swiss primary care active monitoring). Neuchâtel: Observatoire suisse de la santé, 2016.

9 Merçay C. Médecins de premier recours - situation en Suisse tendances récentes et comparaison internationale. Neuchâtel: Observatoire suisse de la santé, 2015.

10 Seematter-Bagnoud LJJ, Jaccard Ruedin H, Roth M, et al. Offre et recours aux soins médicaux ambulatoires en Suisse - Projections l'horizon 2030, 2008.

11 Ochs NCJ, Senn N. Médecine de famille et coordination des soins: revue de littérature et perspectives pour Le Canton de Vaud. service de la santé publique Du Canton de Vaud, 2015.

12 Peters DH, Adam T, Alonge O, et al. Implementation research: what it is and how to do it. BMJ 2013;347:f6753.

13 Smith P, Mossialos E, Papanicolas I, et al. Performance measurement for health system improvement experiences. Cambridge: Challenges and Prospects, 2009.

14 Connelly JB. Evaluating complex public health interventions: theory, methods and scope of realist enquiry. J Eval Clin Pract 2007;13:935-41.

15 Greenhalgh T, Papoutsi C. Studying complexity in health services research: desperately seeking an overdue paradigm shift. BMC Med 2018;16:95.

16 Marchal B, van Belle S, van Olmen J, et al. Is realist evaluation keeping its promise? A review of published empirical studies in the field of health systems research. Evaluation 2012;18:192-212.

17 Pawson R. Simple principies for the evaluation of complex programmes. CIDADES, Comunidades e Territórios 2004;8:95-107.

18 Flynn R, Rotter T, Hartfield D, et al. A realist evaluation to identify contexts and mechanisms that enabled and hindered implementation and had an effect on sustainability of a lean intervention in pediatric healthcare. BMC Health Serv Res 2019;19:912.

19 Rycroft-Malone J, Fontenla M, Bick D, et al. A realistic evaluation: the case of protocol-based care. Implement Sci 2010;5:38.

20 van Reedt Dortland AKB, Peters LL, Boenink AD, et al. Assessment of biopsychosocial complexity and health care needs: measurement properties of the INTERMED self-assessment version. Psychosom Med 2017;79:485-92.

21 Huyse FJ, Lyons JS, Stiefel FC, et al. 'INTERMED': a method to assess health service needs. Gen Hosp Psychiatry 1999;21:39-48. 
22 Stiefel FC, de Jonge P, Huyse FJ, et al. 'INTERMED': a method to assess health service needs. II. Results on its validity and clinical use. Gen Hosp Psychiatry 1999;21:49-56.

23 Europe WROf. Integrated care models: an overview Copenhagen: who regional office for Europe, 2016.

24 Proctor E, Silmere H, Raghavan R, et al. Outcomes for implementation research: conceptual distinctions, measurement challenges, and research agenda. Adm Policy Ment Health 2011;38:65-76.

25 REDCap, 2019. Available: https://www.project-redcap.org/

26 Thomas DR. A general inductive approach for analyzing qualitative evaluation data. Am J Evaluat 2006;27:237-46.

27 Blais MMS. L'analyse inductive générale: description d'une démarche visant donner un sens des données brutes. Recherche Qualitative 2006:1-18.

28 Creswell JW, Fetters MD, Ivankova NV. Designing a mixed methods study in primary care. Ann Fam Med 2004;2:7-12.

29 Oostenbrink JB, Koopmanschap MA, Rutten FFH. Standardisation of costs: the Dutch manual for costing in economic evaluations. Pharmacoeconomics 2002;20:443-54.

30 Basu S, Landon BE, Williams JW, et al. Behavioral health integration into primary care: a Microsimulation of financial implications for practices. J Gen Intern Med 2017;32:1330-41.

31 Beaulieu M-D, Dragieva N, Del Grande C, et al. The team climate inventory as a measure of primary care teams' processes: validation of the French version. Healthc Policy 2014;9:40-54.

32 Warr P, Cook J, Wall T. Scales for the measurement of some work attitudes and aspects of psychological well-being. J Occupat Psychol 1979;52:129-48.

33 Levesque J-F, Pineault R, Provost S, et al. Assessing the evolution of primary healthcare organizations and their performance (2005-2010) in two regions of Québec Province: Montréal and Montérégie. BMC Fam Pract 2010;11:95.

34 Joober H, Chouinard M-C, King J, et al. The patient experience of integrated care scale: a validation study among patients with chronic conditions seen in primary care. Int J Integr Care 2018;18:1.

35 Etz RS, Zyzanski SJ, Gonzalez MM, et al. A new comprehensive measure of high-value aspects of primary care. Ann Fam Med 2019;17:221-30.

36 Pluye P, Hong QN. Combining the power of stories and the power of numbers: mixed methods research and mixed studies reviews. Annu Rev Public Health 2014;35:29-45.

37 Pawson R, Tilley N. Realistic evaluation. London: Sage, 1997.

38 Wong G, Westhorp G, Manzano A, et al. RAMESES II reporting standards for realist evaluations. BMC Med 2016;14:96.

39 Hudon C, Chouinard M-C, Aubrey-Bassler K, et al. Case management in primary care for frequent users of healthcare services with chronic diseases and complex care needs: an implementation and realist evaluation protocol. BMJ Open 2018;8:e026433

40 Currie J, Chiarella M, Buckley T. Privately practicing nurse practitioner services in Australia and patient access to care: results from realist interviews. J Am Assoc Nurse Pract 2018;30:344-53.

41 Manzano A. The craft of interviewing in realist evaluation. Evaluation 2016;22:342-60.

42 Moullin JC, Sabater-Hernández D, Benrimoj SI. Model for the evaluation of implementation programs and professional pharmacy services. Res Social Adm Pharm 2016;12:515-22.

43 Hudon C, Chouinard M-C, Aubrey-Bassler K, et al. Case management in primary care among frequent users of healthcare services with chronic conditions: protocol of a realist synthesis. BMJ Open 2017; 7:e017701. 